\title{
The Impact of Device Associations on Human Memory Performance
}

\author{
Madeleine Steeds \\ Department of Computer Science \\ University of Manchester \\ Manchester, UK \\ madeleine.steeds@manchester.ac.uk
}

\begin{abstract}
This project investigates whether associations (or stereotypes) connected to technological devices impact human memory, i.e do people remember more when using one device over another? To do this the project aims to investigate (1) the associations made with devices (e.g. utilitarian or hedonic qualities); (2) whether these associations impact human memory in controlled settings; and (3) if these effects transfer to applied settings. This paper describes the progress made so far in addressing Aim 1 and steps currently being undertaken to address Aim 2. The paper further identifies areas of interest surrounding Aim 3.
\end{abstract}

Human Memory, Cognition, Device Use, Device Stereotypes

\section{TOPIC AND MOTIVATION}

This $\mathrm{PhD}$ research project is centred around the impact of technology on human memory. Findings from Sparrow et al. (2011) suggest that technology may be negatively impacting memory performance, with individuals remembering where information is rather than the information itself. The extent to which technology may be negatively affecting human memory is not yet known. As such, the project applies established psychological theories to the use of technology, with a particular focus on the role of device associations and stereotypes, to investigate if and how technology may be impacting memory.

\subsection{Related work}

Environmental consistency at encoding (the memorisation of information) and retrieval (remembering previously memorised information) has been the subject of many studies and reviews (Smith and Vela, 2001). Previous research has found that environmental changes between these time points can cause memory performance to worsen, an effect known as environmental context dependency. These can be large differences within an environment (e.g. being underwater or on land; Godden and Baddeley, 1975) or relatively small differences (such as odour; Herz, 1997). Wright and Shea (1991) found that inconsistency in factors such as where information was placed on a computer screen led to greater errors in retrieval. However, it has yet to be investigated whether using one device at encoding (e.g. a desktop) and a different device at retrieval (e.g. a smartphone) has an impact on memory performance.

Some comparisons between devices have been made beyond the area of environmental context dependency. Heo (2003) found that memory for content showed on large screens was better than memory for content on small screens. However, Kelley (2007) found that screen size had no effect on learning the content of news stories, contradicting earlier findings. A dissertation by Holdener (2008) investigated whether smartphones or desktop computers led to greater performance in memory for different media types. Those using a smartphone performed better in the memory measure, further contradicting earlier findings of screen size and suggesting the device itself may be an influencing factor. However, this study was conducted in 2008 and it could be a case that the smartphone used in the study was relatively novel, as the original iPhone was released in summer 2007. Therefore, participants may have attended to it more than the desktop, causing improved performance in memory tasks. Overall, the findings of these studies suggest more research may be needed to further investigate which factors surrounding a device impact memory performance. 
The choice of device has also been found to impact cognitive abilities beyond memory. Liu and Wang (2016) investigated whether using tablets or desktop computers impacted decision making. Participants read descriptions of two hotels and chose which one they would book in a hypothetical scenario. One hotel was a hedonic choice while the other was more utilitarian. Participants were also asked to rate the extent they felt tablets and desktops were utilitarian or hedonic. It was found that those on desktops tended to choose the utilitarian hotel while those on tablets chose the hedonic option more frequently. It was further found that those with stronger perceptions of device stereotypes, were more likely to choose a hotel in keeping with the device stereotype suggesting that perceptions of a device can impact decision making.

Furthermore, it has been found that mobile technology can be placed in the Stereotype Content Model (SCM). The SCM has been used in psychology to assess how a group is perceived along 2 axes, competence and warmth (Fiske et al., 2002). When applied to people using mobile devices, Schwind et al. (2019) found that people using smartphones were seen as more contemptuous than those using other, similar devices (e.g. tablets). This suggests that the device itself carries stereotypes that are consistent with stereotypes pertaining to humans. Given this, there is space for future research to investigate the role of these device stereotypes in interaction behaviour.

\subsection{The Project}

Memory loss can become problematic and interrupt daily life and as such it is important that elements which may impact memory performance are researched to help prevent memory loss where possible. The evidence presented in 1.1 suggests that technology may be one of these elements, but current understanding of why memory degradation can occur is limited. Understanding how technology degrades memory may also give insight into ways it can be used to improve memory. As such the present research aims to investigate one way technology may impact memory and assess it in theoretical and applied settings.

The main research questions are:

1. What associations do people make with devices?

2. Do these associations affect human memory in controlled settings?

3. Do these effects occur in real life contexts?

\section{PROJECT PROGRESS}

\subsection{Approach}

This project is made of multiple studies and utilises a mix of methodologies. To address question 1, the project began with an online survey investigating associations made with devices and whether a person's use of devices impacts these. Following this, an experimental approach is planned to investigate whether associations impact human memory in a heavily controlled environment. A similar experimental design will then be applied to more ecologically valid scenarios, with adaptations to suit each application, to investigate whether the effects persist in real world contexts.

\subsection{Completed Work}

So far the associations individuals make with desktop computers, smartphones, laptops and tablets has been investigated using three metrics. The first investigated the hedonic and utilitarian properties of the devices using the scale by Batra and Ahtola (1991). The results suggested that smartphones were seen as significantly more hedonic than the other devices. Similarly, tablets were seen as less utilitarian than the other devices.

The second measure of associations used the SCM in a partial replication of the study by Schwind et al. (2019). Their study only looked at mobile devices and so the overlap with the present work related to only smartphones and tablets, and novelly looked at the associations made with desktops and laptops. The results suggested there were no significant differences between the devices, indicating that the stereotypes held about devices may not always be consistent.

The final measure of perceptions was a free association task, where participants were shown the name of a device and asked to enter as many words as they related to that device. For this measure, only desktops and smartphones were investigated. Participants were shown the name of the device and asked to type in words they associated with it. Participants generated 813 different words relating to desktops and 645 relating to smartphones. The words give insight into what people associate with the devices, and are being utilised as stimuli in the ongoing work.

\subsection{Ongoing Work}

The lab-based memory study was planned, however due to COVID-19, this has been put on hold indefinitely. Instead an online Stroop task (Stroop, 1935) is being run to investigate whether the previously identified device stereotypes cause 
interference when processing word colours. It is hypothesised that words congruent with the device being used will result in faster response times than words incongruent with the device being used.

In parallel to this study being run, a systematic review is being conducted. This review aims to investigate the work comparing device effects on memory and in particular, identify which types of memory (e.g short-term, spatial) are impacted. This will hopefully give insight into domains where the effects of device changes have been investigated and/or applied, and where there are gaps for future research.

\subsection{Future Work}

The next step of the project will be to investigate the effects of device associations on memory in applied contexts. Proposed contexts for consideration are education and media consumption. Each of these contexts provides insight into real world problems but they each come with their own challenges as well.

Learning involves committing information to memory (Oxford English Dictionary, 2009) thus educational contexts are a natural place to apply memory research, and one metric of how much has been learnt is performance in examinations. However, for researchers there are many ethical considerations associated with this. It is not ethical to test half a cohort on one device and half on another, if the hypothesis is that one group performs better, as this is purposefully impeding students academic progress. However, creating fake examinations will be less ecologically valid, and participants may not try as hard to learn the test content and thus may underperform. Therefore, while a pertinent area to apply memory research, it is not straightforward to apply it in an ecologically valid and ethical way.

Media consumption is an interesting topic for applying this research, as the rate of media consumption has slowly increased over the past decade (Statista, 2019) and it is possible to consume this media on a variety of devices. However, to ask a person to watch television in a lab setting lacks ecological validity, but having participants watch content at home means an array of distractions may be present which could hinder memory performance. Furthermore, while exploring genres of media may lend itself to investigating the impact of stereotypes, individual preferences for content may cause large variations in memory performance.

Given these concerns, the specific application areas this project will investigate is still uncertain.

\section{CONTRIBUTION}

The project aims to give insight into the associations made with devices and how these associations may impact device interaction in terms of memory effects. The stereotype findings from the work conducted so far contributes towards understanding how people see their devices. The findings of how the investigated devices are perceived (e.g. the free association data) can be used as a resource in future research, for example how this project will use the free association data as stimuli.

The findings from the ongoing and future work may have implications for health in terms of giving insight into whether device associations play a role in memory degradation and ways to mitigate these issues by using the effect of associations to the users advantage. Depending on the direction of the applied work, this may also benefit other sectors such as education or media companies.

\section{REFERENCES}

Batra, R. and O. T. Ahtola (1991). Measuring the hedonic and utilitarian sources of consumer attitudes. Marketing letters 2(2), 159-170.

Fiske, S. T., A. J. Cuddy, P. Glick, and J. Xu (2002). A model of (often mixed) stereotype content: competence and warmth respectively follow from perceived status and competition. Journal of personality and social psychology 82(6), 878.

Godden, D. R. and A. D. Baddeley (1975). Contextdependent memory in two natural environments: On land and underwater. British Journal of psychology 66(3), 325-331.

Heo, N. (2003). The effects of screen size and content type on viewers' attention, arousal, memory, and content evaluations.

Herz, R. S. (1997). The effects of cue distinctiveness on odor-based context-dependent memory. Memory \& Cognition 25(3), 375-380.

Holdener, A. (2008). Does size matter?

Kelley, M. (2007). The effect of screen size and audio delivery system on memory for television news. Visual Communication Quarterly 14(3), 176-188.

Liu, Y. and D. Wang (2016). How does the device change your choice: A goal-activation perspective. In International Conference on $\mathrm{HCl}$ in Business, Government, and Organizations, pp. 446-456. Springer.

Oxford English Dictionary (2009). Oxford English Dictionary. Oxford: Oxford University Press. 
Schwind, V., N. Deierlein, R. Poguntke, and $\mathrm{N}$. Henze (2019). Understanding the social acceptability of mobile devices using the stereotype content model. In Proceedings of the $2019 \mathrm{CHI}$ Conference on Human Factors in Computing Systems, pp. 1-12.

Smith, S. M. and E. Vela (2001). Environmental context-dependent memory: A review and metaanalysis. Psychonomic bulletin \& review 8(2), 203-220.

Sparrow, B., J. Liu, and D. M. Wegner (2011). Google effects on memory: Cognitive consequences of having information at our fingertips. science 333(6043), 776-778.

Statista (2019). Time spent with media worldwide 2018.

Stroop, J. R. (1935). Studies of interference in serial verbal reactions. Journal of experimental psychology 18(6), 643.

Wright, D. L. and C. H. Shea (1991). Contextual dependencies in motor skills. Memory \& Cognition 19(4), 361-370. 